\title{
Marjolin's ulcers at a university teaching hospital in Northwestern Tanzania: a retrospective review of 56 cases
}

Phillipo L Chalya ${ }^{1 *}$, Joseph B Mabula $1^{1+}$, Peter Rambau ${ }^{2+}$, Mabula D Mchembe ${ }^{4 \dagger}$, Kahima J Kahima ${ }^{2 \dagger}$, Alphonce B Chandika ${ }^{1 \dagger}$, Geofrey Giiti ${ }^{1+}$, Nestory Masalu ${ }^{3 \dagger}$, Robert Ssentongo ${ }^{5+}$ and Japhet M Gilyoma ${ }^{1+}$

\begin{abstract}
Background: Marjolin's ulcer is a rare but highly aggressive squamous cell cancer that is most often associated with chronic burn wounds. Although many individual case reports exist, no comprehensive evaluation of Marjolin's ulcer patients has been conducted in our setting. This study was conducted to describe the clinicopathological presentation and treatment outcome of this condition in our local setting and to identify predictors of outcome.

Methods: This was a retrospective study of histologically confirmed cases of Marjolin's ulcer seen at Bugando Medical Centre over a period of 10-years between January 2001 and December 2010. Data were retrieved from patients' files and analyzed using SPSS computer software version 15.0

Results: A total of 56 patients were studied. Male to female ratio was 2.1:1. Burn scars (89.3\%) were the most common causative lesions of Marjolin's ulcer. The mean latent period between original injury and diagnosis of Marjolin's ulcer was $11.34 \pm 6.14$ years. Only $12.0 \%$ of the reported cases were grafted at the time of injury $(P<$ 0.00). Most patients (48.2\%) presented between one and five years of onset of illness. The lower limb (42.9\%) was the most frequent site for Marjolin's ulcers. The median tumor size at presentation was $8 \mathrm{~cm}$ and the vast majority of patients (85.7\%) presented with large tumors of $\geq 5 \mathrm{~cm}$ in diameter. Lymph node metastasis at the time of diagnosis was recorded in $32.1 \%$ of cases and distant metastasis accounted for $26.9 \%$ of cases. Squamous cell carcinoma (91.1\%) was the most common histopathological type. Wide local excision was the most common surgical procedure performed in $80.8 \%$ of cases. Post-operative complication rate was $32.1 \%$ of which surgical site infection was the most common complication in 38.9\% of patients. Local recurrence was noted in $33.3 \%$ of cases who were treated surgically. The mean length of hospital stay for in-patients was $7.9 \pm 2.3$ days. Mortality rate was $7.1 \%$. According to multivariate logistic regression analysis, stage and grade of the tumor and presence of local recurrence were the main predictors of death $(P<0.001)$.
\end{abstract}

Conclusion: Marjolin's ulcers are not rare in our environment and commonly occur in burn scars that were not skin grafted and were left to heal secondarily. A high index of suspicion is required in the management of chronic non-healing ulcers and all suspected lesions should be biopsed. Early recognition and aggressive treatment of Marjolin's ulcers and close follow-up are urgently needed to improve outcomes in our environment.

Keywords: Marjolin?'?s ulcers, clinicopathological pattern, treatment outcome, Tanzania

\footnotetext{
* Correspondence: drphillipoleo@yahoo.com

+ Contributed equally

'Department of Surgery, Catholic University of Health and Allied Sciences-

Bugando, Mwanza, Tanzania

Full list of author information is available at the end of the article
} 


\section{Background}

Marjolin's ulcer is a rare and often aggressive cutaneous malignancy that arises in previously traumatized or chronically inflamed skin, particularly after burns [1]. Marjolin's ulcers have a $1 \%$ to $2 \%$ incidence in all burn scars but can also develop from previously traumatized and scarred tissue of other etiologies such as chronic sinuses of osteomyelitis, post-traumatic wounds, pressure sores and chronic fistulae and have been reported to arise in the genitalia as a complication of Fournier's gangrene [2]. The term "Marjolin's ulcer" was named after French surgeon, Jean Nicolas Marjolin, who first described the condition in 1828 [1-3]. But it was Dupuytren who noted it was a malignancy [4]. In 1923, DaCosta first coined the expression "Marjolin's ulcer" to describe malignant tumors forming over burn injuries [5].

The exact mechanism of malignant transformation of Marjolin's ulcer remains unclear and controversial [6-8]. Several theories including the toxin, chronic irritation, traumatic epithelial elements implantation, heredity, immunologic privileged site, co-carcinogen, ultraviolet rays, initiation and promotion and environmental and genetic interaction theories have been reported to explain the malignant transformation [9].

The latency period from the time of injury to the onset of malignant transformation averages 36 years [10]. However, early arising Marjolin's ulcers have been described in the literature $[8,11]$. Studies from western countries have shown that the average age at diagnosis is in the fifth decade of life with a range of 18-84 years, and men are three times more frequently affected than women [11]. In Sub-Saharan Africa, Marjolin's ulcer appears to be affecting younger patients and the transition time is getting shorter over the years [12].

Most lesions of Marjolin's ulcer occur on the extremities $(60 \%)$, with ulcers on the head and face occurring less frequently $(30 \%)$ and the lowest frequency $(10 \%)$ on the trunk [4]. Marjolin's ulcers in unusual sites such as the genitalia as a complication of Fournier's gangrene [13] and breast skin developing in a postburn scar [14] have also been reported.

Marjolin's ulcers are very aggressive tumors that necessitate a well thought out treatment plan to optimize care and assure patient survival [14]. Early diagnosis and prompt surgical intervention is mandatory in Marjolin's ulcers as they may invade vital structures. However, in developing countries like Tanzania, most patients with these lesions are treated as chronic ulcers or infections and present late to hospitals, leading to a delayed diagnosis and resulting in the need for more extensive surgery and an increased risk of metastasis.

Marjolin's ulcers presents histopathologically as squamous cell carcinoma in $75 \%$ to $96 \%$ of cases [15]. Other neoplasms, such as basal cell carcinomas, melanoma, osteogenic sarcoma, fibrosarcoma, and liposarcoma, have also been reported $[8,16]$.

In spite of being a surface malignancy and more amenable not only to early detection, but also to a potential cure, the outcome of treatment in most developing countries including Tanzania has been poor because the majority of these patients present late to the hospital with advanced stage. This is partly due to paucity of local data regarding this condition and lack of community awareness on the importance of early reporting to hospital for early diagnosis and treatment. This study was conducted to evaluate the clinicopathological presentation and treatment outcome of this condition in our local setting and to identify predictors of outcome.

\section{Methods \\ Study design and setting}

This was a retrospective study of histologically confirmed cases of Marjolin's ulcer seen at the department of Surgery of Bugando Medical Centre (BMC) over a period of 10-years between January 2001 and December 2010. BMC is a consultant, tertiary care and teaching hospital for the Catholic University of Health and allied Sciences-Bugando (CUHAS-Bugando) and has a bed capacity of 1000 .

\section{Study population}

The subjects of this study included all patients who presented to BMC with histologically confirmed Marjolin's ulcers during the period studied. Patients with incomplete data were excluded from the study. The details of patients were retrieved from patients' files kept in the Medical record department, the surgical wards, operating theatre and histopathology laboratory. Information retrieved included demographic data, causative previous lesions, latency period, duration of illness, anatomical site, radiological investigations, histological types, treatment modalities, management complications, length of hospital stay and mortality.

\section{Statistical analysis}

Data collected were analyzed using SPSS computer software version 15.0 (SPSS, Inc, Chicago, IL). Data was summarized in form of proportions and frequency tables for categorical variables. Continuous variables were summarized using mean, median, mode and standard deviation. Chi -square $\left(\chi^{2}\right.$-test $)$ was used to test for significance of associations between the predictor and outcome variables in the categorical variables. Student $t-$ test was used to test for significance of associations between the predictor and outcome variables in the 
continuous variables. Significance was defined as a pvalue of $<0.05$. Multivariate logistic regression analysis was used to determine predictor variables that are associated with outcome.

\section{Ethical consideration}

Ethical approval to conduct the study was sought from the CUHAS-Bugando/BMC joint institutional ethic review committee before the commencement of the study.

\section{Results}

\section{Demographic data}

During the period under study, a total of 56 patients were studied. Males were $38(67.9 \%)$ and females were $18(32.1 \%)$ with a male to female ratio of $2.1: 1$. Their mean and median ages were $38.2 \pm 24.2$ years and 37 years respectively (range 12-78 years). The modal age group was 31-40 years. The majority of patients, 39 (69.6\%) were younger than 40 years. The vast majority of patients, $49(87.5 \%)$ came from the rural areas located a considerable distance from Mwanza City and most of them, 51 (91.1\%) had either primary or no formal education.

\section{Causative previous lesions}

Burn scars were the most common causative lesions of Marjolin's ulcer, representing $89.3 \%$ of cases. The mean latent period between original injury and development of Marjolin's ulcer was $11.34 \pm 6.14$ years (range from five months to 48 years). Skin grafting of the previous causative lesions was reported in only six (10.7\%) patients and the rest of the lesions healed by either delayed primary or secondary intention.

\section{Clinical presentation}

The duration of illness (the period from development of Marjolin's ulcer and presentation to health facility) ranged from 1 month to 8 years with a median of 4 years and the majority of patients (48.2\%) presented between one and five years of onset of illness. The reasons for late presentation are shown in Table 1. Forty-six (82.1\%) patients had undergone some form of intervention in peripheral dispensaries and hospitals before been referred, with dressing and inadequate surgical resection being the most common intervention.

The lower limb was the most frequent site for Marjolin's ulcers accounting for $42.9 \%$ of patients. The majority of patients presented with increasing pain $(92.9 \%)$ and foul smelling pus discharge $(89.3 \%)$ and most of them (51.8\%) had exophytic proliferative ulcers. The median tumor size at presentation was $8 \mathrm{~cm}$ (range 2 to $16 \mathrm{~cm}$ ) and the vast majority of patients $(85.7 \%)$ presented with large tumors of $\geq 2 \mathrm{~cm}$ in diameter. Most patients (44.6\%) had no evidence of metastases. Lymph node metastasis at the time of diagnosis was recorded in $32.1 \%$ of cases. Distant metastasis accounted for $26.9 \%$ of cases and occurred mostly in the lungs, liver, bone and brain in $6(40.0 \%), 5(33.3 \%), 2(13.3 \%)$ and 2 (13.3\%) patients respectively (Table 1 ).

\section{Investigations}

A total of 70 radiological investigations were performed to rule out distant metastasis; of these, plain radiographs were the most frequent radiological investigation performed accounting for $64.3 \%$ of all the radiological investigations (Table 2). Table 3 shows histopathological findings of various Marjolin's ulcers. Squamous cell carcinoma was the most common histopathological type accounting for $91.1 \%$ of all Marjolin's ulcers. The majority of these tumors (Marjolin's ulcers) were moderate differentiation in $41.1 \%$. The depth of dermal invasion and vertical tumor thickness is shown in Table 3.

\section{Treatment modalities}

In the present study, fifty-two (92.9\%) patients underwent surgical treatment. Of these, wide local excision was the most common surgical procedure performed in $80.8 \%$ of cases (Table 4 ).

\section{Clinical Outcome}

Post-operative complications were recorded in 18 (32.1\%) patients. Of these, surgical site infection was the most common post-operative complication accounting for $38.9 \%$ of cases (Table 5). The majority of patients, 51 (91.1\%) required hospitalization for their treatment and the remaining $5(8.9 \%)$ were treated as outpatients. The length of hospital stay for in-patients ranged from 6 to 21 days (mean $7.9 \pm 2.3$ days). Four patients died in hospital giving a mortality rate of $7.1 \%$. According to multivariate logistic regression analysis, stage and grade of the tumor, presence of metastases and presence of local recurrence were the main predictors of death $(\mathrm{P}<$ 0.001 ). Local recurrence was noted in $33.3 \%$ of cases who had surgical treatment. All cases of local recurrence of ulcers occurred from 6 months to 1 year (mean $8.3 \pm 3.4$ months) after definitive treatment. Tumor size, histological grade of the tumor, stage of the tumor and presence of metastasis at the time of diagnosis were the main predictors of local recurrence $(\mathrm{P}<0.001)$.

\section{Discussion}

Marjolin's ulcer is defined as a tumor arising from a chronic wound, scar or chronic inflammation [1,2]. Jean Nicholas Marjolin first described the malignant transformation of cutaneous scars in 1828 [1-3]. Since then, several reports of post-burn scar ulcers have been reported 
Table 1 Clinical presentation

\begin{tabular}{|c|c|c|c|}
\hline Variables & Responses & Frequency & Percentage \\
\hline \multirow[t]{7}{*}{ Previous lesions } & Burn scars & 50 & 89.3 \\
\hline & Penile human bite scar & 1 & 1.8 \\
\hline & Pressure sores & 1 & 1.8 \\
\hline & Chronic non healing ulcer & 1 & 1.8 \\
\hline & Chronic osteomyelitis & 1 & 1.8 \\
\hline & Diabetic foot ulcer & 1 & 1.8 \\
\hline & Fournier's gangrene & 1 & 1.8 \\
\hline \multirow[t]{3}{*}{ Duration of illness (years) } & $<1$ & 13 & 23.2 \\
\hline & $1-5$ & 27 & 48.2 \\
\hline & $>5$ & 16 & 28.6 \\
\hline \multirow[t]{6}{*}{ Reason for late presentation } & Financial problem & 34 & 60.7 \\
\hline & Treated at peripheral hospitals & 32 & 57.1 \\
\hline & Treated by traditional healers & 31 & 55.4 \\
\hline & Lack of money for transport & 31 & 55.4 \\
\hline & Self medication at home & 12 & 21.4 \\
\hline & No reasons documented & 12 & 21.4 \\
\hline \multirow{5}{*}{ Site of the lesion } & Lower limb & 24 & 42.9 \\
\hline & Head \& neck & 13 & 23.2 \\
\hline & Upper limb & 12 & 21.4 \\
\hline & Trunk & 4 & 7.1 \\
\hline & Genitalia & 2 & 3.6 \\
\hline \multirow[t]{5}{*}{ Symptoms } & Increasing pain & 52 & 92.9 \\
\hline & Foul smelling pus discharge & 50 & 89.3 \\
\hline & Haemorrhage & 36 & 64.3 \\
\hline & Increasing discharge & 35 & 62.5 \\
\hline & Increasing tumor size & 30 & 53.6 \\
\hline \multirow[t]{2}{*}{ Tumor type } & Exophylic & 27 & 48.2 \\
\hline & Infiltrative & 29 & 51.8 \\
\hline \multirow[t]{2}{*}{ Tumor size } & Less than $5 \mathrm{~cm}$ diameter & 8 & 14.3 \\
\hline & $5 \mathrm{~cm}$ or more diameter & 48 & 85.7 \\
\hline \multirow[t]{3}{*}{ Metastasis at the time of diagnosis } & Lymph node metastasis & 18 & 26.9 \\
\hline & Distant metastasis & 15 & 32.1 \\
\hline & No evidence of metastasis & 25 & 44.6 \\
\hline
\end{tabular}

[7,8,11]. Various studies indicate that Marjolin's ulcers make up $1.2 \%$ of all skin cancers $[7,17]$.

In this review, the mean age of patients was 38.2 years which is lower than the age reported in western countries $[11,18,19]$. Current studies in Africa have reported that the mean age of patients with Marjolin's ulcers is lowering and appears to be affecting younger patients

Table 2 Radiological investigations ( $N=70$ )

\begin{tabular}{lll}
\hline Radiological study & Frequency & Percentage \\
\hline Plain radiographs & $\mathbf{4 5}$ & $\mathbf{6 4 . 3}$ \\
Long bone x-rays & 28 & 62.2 \\
Chest x-rays & 18 & 40.0 \\
Skull x-rays & 12 & 26.7 \\
Abdominal ultrasound & $\mathbf{1 6}$ & $\mathbf{2 2 . 9}$ \\
Computed Tomography (CT) scan brain & $\mathbf{9}$ & $\mathbf{1 2 . 9}$ \\
\hline
\end{tabular}

over the years $[12,20]$. It also appears that the transition time is getting shorter [20]. Marjolin's ulcers in general, develop in younger patients amongst sub-Saharan patients than those reported from other regions [12]; therefore, patients presenting with chronic ulcers should be investigated during the initial evaluation for this possibility. Further research is needed in our region to explain this observation.

The two-fold increase in the number of males with Marjolin's ulcers compared to females in the present study is similar to what was reported in other studies $[7,21,22]$. Male preponderance in the present study may be due to their increased susceptibility to trauma which, if poorly managed, these lesions have been reported to undergo malignant transformation.

Marjolin's ulcer is a malignant change in a long-standing ulcer and/or scar tissue [22,23]. In agreement with 
Table 3 Histopathological results $(\mathrm{N}=56)$

\begin{tabular}{llll}
\hline Variable & Response & Frequency & Percentage \\
\hline $\begin{array}{l}\text { Histopathological } \\
\text { type }\end{array}$ & $\begin{array}{l}\text { Squamous cell } \\
\text { carcinoma }\end{array}$ & 51 & 91.1 \\
& $\begin{array}{l}\text { Basal cell carcinoma } \\
\text { Osteogenic sarcoma }\end{array}$ & 2 & 3.6 \\
& Fibrosarcoma & 1 & 1.8 \\
& Melanoma & 1 & 1.8 \\
& Well differentiated & 16 & 28.6 \\
Tumor grade & $\begin{array}{l}\text { Moderate } \\
\text { differentiated }\end{array}$ & 23 & 41.1 \\
& Poor differentiated & 17 & 30.3 \\
Depth of dermal & Superficial to reticular & 4 & 7.1 \\
invasion & dermis & & \\
& Reticular dermis or & 36 & 64.3 \\
& deep & & \\
Vertical tumor & Lot documented & 16 & 28.6 \\
thickness & Less than 4 mm thick & 9 & 16.1 \\
& 4 mm thick or more & 35 & 62.5 \\
& Not documented & 12 & 21.4 \\
\hline
\end{tabular}

other studies $[4,7,11,14,16]$, the most frequent predisposing lesion of Marjolin's ulcers in the present study was post burn scars. Marjolin ulcers generally occur in regions of previous deep burn that healed slowly without skin grafting [10]. This observation is reflected in our study where only $12.0 \%$ of patients reported to have skin grafting for their previous causative lesions. Burn scar carcinoma has a propensity for the extremities, specifically to flexion creases of the extremities, where blood supply is decreased and vulnerability to trauma is increased [23]. Marjolin's ulcers have also been reported following other traumatic injuries, leg ulceration, chronic sinuses of osteomyelitis, pressure sores, and discoid lupus erythematosus [24]. They have also been documented in the genitalia as a complication of Fournier's gangrene [13]. Interestingly, one patient in our study was a rare case of a 33 year-old patient who presented with an early appearance of Marjolin's ulcer developing in a penile human bite scar seven months after the initial injury.

Table 4 Type of surgical procedures performed $(\mathbf{N}=52)$

\begin{tabular}{lll}
\hline Type of surgical procedure & Frequency & Percentage \\
\hline Wide local excision & $\mathbf{4 2}$ & $\mathbf{8 0 . 8}$ \\
With skin grafting & 36 & 85.7 \\
With flap & 6 & 14.3 \\
Limb amputation & $\mathbf{6}$ & $\mathbf{1 1 . 5}$ \\
Minor & 2 & 33.3 \\
Major & 4 & 66.7 \\
Lymph node dissection & $\mathbf{4}$ & $\mathbf{7 . 7}$ \\
\hline
\end{tabular}

Table 5 Postoperative complications ( $N=18$ )

\begin{tabular}{lll}
\hline Postoperative complications & Frequency & Percentage \\
\hline Surgical site infection & 7 & 38.9 \\
Local recurrence & 6 & 33.3 \\
Loss of skin grafting & 2 & 11.1 \\
Loss of flaps & 1 & 5.5 \\
Stump dehiscence & 1 & 5.5 \\
Re-amputation & 1 & 5.5 \\
\hline
\end{tabular}

The precise mechanism by which chronic ulcers (wounds) develop malignancy is not known and many theories have been postulated [9]. Early theories suggested that cellular mutations as a result of inflammatory related substances release by damaged, ischemic and nutritionally deficient tissues are responsible for neoplastic change [4]. Neuman et al [25] proposed that traumatic displacement of living epithelial tissue into dermis may cause a foreign body response and lead to a deranged regenerative process, resulting in carcinomatous change. More recently, a theory of immunologic isolation has been suggested, whereby lymphatic channel obliteration at the site of injury decreases the delivery of antigen or specifically stimulated small lymphocytes to the regional lymphocytes from that site. This renders the site 'immulogically unprivileged', allowing the development of antigenically foreign tumor cells to go unchecked. Such cells may initially arise by spontaneous mutation or develop under the influence of viral or chemical carcinogens. Tumor antigen recognition may then be delayed long enough for tumors to reach 'critical size', when immune mechanisms are no longer sufficient to prevent continued neoplastic progression [26]. It has also been suggested that patients with an inherent immune deficiency are at higher risk for developing malignant ulcers [27]. Some authors have also postulated that with chronic irritation and repeated damage of the ulcer, there is continuous mitotic activity as the epidermal cells attempt to resurface the open defect. This cycle of damage, irritation, and repair can lead to a malignant transformation [28]. More recent theories have included genetic postulations involving human leukocyte antigen (HLA) DR4 and mutations in the p53 and/or Fas genes [29-32].

Two variants of Marjolin's ulcer have been described; an acute form, in which the cancer occurs within one year of the injury and a chronic form in which malignant changes are more than one year from the date of injury. The chronic form is more frequent and malignancy tends to develop slowly, with an average latency period of 36 years $[21,33]$. The younger the patient at the time of injury, the longer the interval for malignant change, while the older the patient at the time of burning, the longer the lag period. The results of this study 
showed an average latency period of 11 years which is lower than the latency period reported in most western studies $[9,22]$. It has been observed that the latency period is inversely proportional to the patients' age. The reason for this phenomenon is unknown.

In keeping with other studies $[4,18,21,24,34,35]$, the lower limb was the most frequent site for Marjolin's ulcers. The anatomical locations reported by Arons [36], Lawrence [37], and Novick [38] show that average distribution of Marjolin ulcers is $40 \%$ in the lower extremity, $30 \%$ in the head and face, $20 \%$ in the upper extremity, and $10 \%$ in the trunk area. The reason for this anatomical site predilection is not well understood.

Marjolin's ulcers are commonly mistaken for an infected ulceration occurring at the scar tissue sites and may often be overlooked. Changes such as the appearance of flat, non-healing ulcers enlarging in circumference with elevated and indurated borders, foul-smelling, painful with exudates and bloody drainage suggest a malignant transformation $[39,40]$. As in other studies $[8,9,22,39,40]$, the majority of our patients in the present study presented with increasing pain, foul smelling pus discharge, increasing tumor size and most of them had exophytic proliferative ulcers. It is therefore recommended that all chronic ulcers should be thoroughly investigated at presentation, to avoid labeling malignancies 'chronic ulcers', leading to delay in appropriate treatment. All patients presenting with chronic ulcers should undergo multiple biopsies, to help confirm the neoplasmatic conversion and to avoid missing malignant ulcers.

Macroscopically, Marjolin's ulcers have been reported to exist in two forms which are of prognostic importance1) Exophytic form characterized by prolonged and relatively benign course and low probability of distant metastasis 2) Infiltrative form characterized by rapid formation of ulceration and worse prognosis and high probability of metastatic spread [22]. Infiltrative Marjolin's ulcers was the most common form in the present study accounting for $51.8 \%$ of cases and this may be responsible for the high rate of metastatic spread in our study.

Marjolin's ulcers have been reported to have an aggressive course and a much greater tendency to metastasize than other types of skin cancer, which makes early diagnosis imperative. Metastasis to the brain, liver, lung, kidney, and distant lymph nodes has been commonly reported $[8,21,34,35,41]$. In the present study, $32.1 \%$ of patients had lymph node metastasis at the time of diagnosis and $26.9 \%$ of cases had distant metastasis to the lungs, liver, bone and brain, the rate which is higher than that reported in other studies $[34,35]$. High lymph node and distant metastasis in our study is due to the fact that most patients in the present study present late when the disease is already in advanced stages. In developing countries like ours, especially in rural areas with poor living conditions most patients are already in advanced stages of disease at the time of diagnosis of Marjolin's ulcer, which has been proven both in the present study and in literature $[42,43]$. In the present study, more than $80 \%$ of patients presented late with large tumor $>2 \mathrm{~cm}$ in diameter. Late presentation is common in most developing countries as a result of poverty, ignorance, and poor referral systems in a relatively expensive health care system devoid of meaningful health insurance [12]. Financial problem and delayed referral to tertiary health care facility were the most common reasons for late presentation in the present study. Health education is highly needed to discourage patients with cutaneous ulcers from presenting late to hospital when the disease is in its advanced stage. Early adequate treatment of all ulcers and scars will certainly reduce the incidence of Marjolin's ulcer in our setting.

The commonest histopathological type of carcinoma arising from Marjolin's ulcer is squamous cell carcinoma, followed by basal cell carcinoma as the second commonest carcinoma $[7,8,10,16,16,18,22,23]$. This finding is in agreement with the present study in which more than $90 \%$ of Marjolin's ulcers were squamous cell carcinoma. Other reported neoplasms are malignant melanoma, osteogenic sarcoma, fibrosarcoma and liposarcoma $[8,16]$.

The treatment of Marjolin's ulcers requires multidisciplinary approach. Treatment modalities of Marjolin's ulcers include wide local excision, block dissection of the regional nodes, amputation in advanced lesions of limbs, radiotherapy and chemotherapy given either as neo or adjuvant therapy [10]. Wide local excision (surgical margin of at least $2 \mathrm{~cm}$ ), together with skin grafting primarily or primarily delayed, is usually considered appropriate in the treatment of Marjolin's ulcers $[10,40]$. Adequate surgical resection is most important to prevent local recurrence and a margin of $2-5 \mathrm{~cm}$ has been advocated $[12,40]$. Frozen sections have been reported to be used for intraoperative diagnosis and evaluation of surgical excision safety margins [17]. However, like in most developing countries, frozen sections are not performed in our centre partly because of few available pathologists and lack of facilities for performing frozen sections.

Amputation is indicated when wide local excision is not possible due to deep invasion, bone or joint involvement, infection, or hemorrhage, or when excision would cause major functional disability. Regional lymph node dissection is indicated when nodes are clinically palpable with an exception for malignant melanoma, where the sentinel lymph node biopsy should be performed 
regardless of the presence of enlarged lymph nodes [40]. Sentinel lymph node biopsy has been shown to give as high yield as $83 \%[40,44]$ and is recommended to detect occult nodal involvement. However, sentinel lymph node biopsy was not performed in the present study due to its unavailability in our centre. In agreement with other studies $[10,40]$, wide local excision with either skin grafting or flap coverage, was the most frequent surgical procedure performed in the present study. Limb amputation and lymph node dissection were performed in $11.5 \%$ and $7.7 \%$ of cases. Radiotherapy and chemotherapy (in the form of four courses of (Methotrexate, Bleomycin and Cisplatinum) is indicated in patients with poor prognostic factors or distant metastasis $[24,36]$. In our study, patients who had inoperable tumor and those who had recurrences were referred to Oncological centre for possible palliative radiotherapy/ chemotherapy.

Most series indicate that the incidence of recurrence is in the range of $20 \%$ to $50 \%$ [16,24]. Most recurrences are regional, but metastases to the brain, liver, lung, kidney, and distant lymph nodes have been reported [24]. In the present study, local recurrence was recorded in $33.3 \%$ of cases which is higher rate than that is reported by other authors $[8,21,34,35,41]$. High recurrence rate in our study is attributed to delayed presentation and diagnosis and this confirmed the highly metastatic potential of Marjolin's ulcer among skin malignancies.

Recurrence and fatality rates are higher due to the aggressive nature of this tumor [28,33].

Our overall mortality rate in the present study was $7.1 \%$ that is relatively lower than that reported in other studies [35,44]. In the present study, mortality rate was significantly high in patients with high stage and grade of the tumor and those who had metastases and local recurrences reflecting the aggressive nature of this tumor.

The prognosis in Marjolin's ulcers depends on various factors like age of the patient, size, grade and stage of the tumor, presence of metastases, the adequacy of surgery and presence of local recurrence. In the present study, stage and grade of the tumor, presence of metastases and presence of local recurrence were the main predictors of death. Appropriate Marjolin's ulcer patient prognostication is of paramount important in clinical decision making, especially the utilization of resources in poor income countries.

\section{Conclusion}

Marjolin's ulcers are not uncommon in our setting and commonly occur in burn scars that were not skin grafted and were left to heal secondarily. They have a shorter latent period and commonly occurred in younger male patients. Most patients in our environment present late when the disease is already in advanced stages. Recurrence rate following surgical treatment was $33.3 \%$. A high index of suspicion is required in the management of chronic non-healing ulcers that are recalcitrant to therapy and all suspected lesions should be biopsed. Early recognition and aggressive treatment of Marjolin's ulcers and close follow-up are urgently needed to improve outcomes in our environment. Health education is highly needed to discourage patients with cutaneous ulcers from presenting late to hospital when the disease is in its advanced stage.

\section{Acknowledgements}

The authors acknowledge all those who provided support in preparation of this manuscript. Special thanks go to the staff members of Medical records department of Bugando Medical Centre for their support during data collection.

\section{Author details}

'Department of Surgery, Catholic University of Health and Allied SciencesBugando, Mwanza, Tanzania. ${ }^{2}$ Department of Pathology, Catholic University of Health and Allied Sciences-Bugando, Mwanza, Tanzania. ${ }^{3}$ Department of Oncology, Catholic University of Health and Allied Sciences-Bugando,

Mwanza, Tanzania. ${ }^{4}$ Department of Surgery, Muhimbili University of Health and Allied Sciences, Dar Es Salaam, Tanzania. ${ }^{5}$ Department of Plastic and Reconstructive Surgery, Mulago Hospital Complex, Kampala, Uganda.

\section{Authors' contributions}

PLC contributed in study design, literature search, data analysis, manuscript writing, editing and submission of the manuscript. JBM, PR, ABC, GG and KJK participated in study design, data analysis, manuscript writing \& editing. MDM participated in data analysis, manuscript writing \& editing. NM and RS participated in data analysis and manuscript writing. JMG supervised the study and contributed in data analysis, manuscript writing \& editing. All the authors read and approved the final manuscript.

\section{Competing interests}

The authors declare that they have no competing interests.

Received: 28 December 2011 Accepted: 15 February 2012

Published: 15 February 2012

\section{References}

1. Sabin SR, Goldstein G, Rosenthal HG, Haynes KK: Aggressive squamous cell carcinoma originating as a Marjolin's ulcer. Dermatologic Surgery 2004, 30:229-30.

2. Duncan KO, Leffell DJ: Epithelial precancerous lesions. In Fitzpatrick's dermatology in general medicine.. 6 edition. Edited by: Freedberg IM, Eisen AZ, Wolff K. New York: Mc Graw-Hill; 2003:719-36.

3. Marjolin NJ: Ulcère. Dictionnaire de medecine 1828, 21:31.

4. Treves N, Pack GT: The development of cancer in burn scars. Surg Gynecol Obstet 1930, 51:749-782.

5. Da Costa JC: Carcinomatous changes in an area of chronic ulceration, or Marjolin's ulcer. Ann Surg 1903, 37:495-502.

6. Tan O, Atik B, Bekerecioglu M, Tercan M, Bayram I: Squamous carcinoma in a pressure sore with a very short latency period. Eur J Plast Surg 2003, 26:360-2.

7. Er-Fan X, Li AO, Shi-ling W, Shao-Yu K, Guang-Xiu C: Burn scar carcinoma: Case reports and review of the literature. Annals of the MBC 1992, 5:2.

8. Thio D, Clarkson JH, Misra A, Srivastava S: Malignant change after 18 months in a lower limb ulcer: Acute Marjolin's ulcer revisited. British Journal of Plastic Surgery 2003, 56:825-8.

9. Nthumba PM: Marjolin's ulcers: theories, prognostic factors and their peculiarities in spina bifida patients. World Journal of Surgical Oncology 2010, 8:108.

10. Ochenduszkiewicz U, Matkowski1 R, Szynglarewicz1 B, Kornafel J: Marjolin's ulcer: malignant neoplasm arising in scars. Rep Pract Oncol Radiother 2006, 11(3):135-138. 
11. Dávalos BA, Cortés-Flores AO, Bandera-Delgado A: Malignant neoplasm in burn scar: Marjolin's ulcer: Report of two cases and review of the literature. Cir Cir 2008, 76:329-31.

12. Nthumba PM: Marjolin's ulcers in sub-Saharan Africa. World J Surg 2010, 34:2272-2277

13. Chintamani M, Shankar M, Singhal V, Singh JP, Bansal A, Saxena S: Squamous cell carcinoma developing in the scar of Fournier's gangrene Case report. BMC Cancer 2004, 274(1):16.

14. Calikapan GT, Akan M, Karaca M, Aköz T: Marjolin ulcer of the scalp: intruder of a burn scar. J Craniofac Surg 2008, 19:1020-1025.

15. Ozek C, Celik N, Bilkay U, Akalin T, Erdem O, Cagdas A: Marjolin's ulcer of the scalp: Report of 5 cases and review of theliterature. J Burn Care Rehabil 2001, 22:65-9.

16. Suhag V, Singh S, Nimbrian VK, Dimri K, Sharma N: Marjolin's Ulcer developing in electrical burns: A rare case report. Pakistan Journal of Medical Science 2005, 21:375-6.

17. Daya M, Balakrishan T: Advanced Marjolin's ulcer of the scalp in a 13year-old boy treated by excision and free tissue transfer: Case report and review of literature. Indian J Plast Surg 2009, 42:106-.

18. Bozkurt M, Kapi E, Kuvat SV, Ozekinci S: Current concepts in the management of Marjolin's ulcers: outcomes from a standardized treatment protocol in 16 cases. J Burn Care Res 2010, 31(5):776-80

19. Fleming MD, Hunt JL, Purdue GF, Sandstad J: Marjolin's ulcer: a review and re-evaluation of a difficult problem. J Burn Care Rehabil 1990, 11(5):460-9.

20. Chlihi A, Bouchta A, Benbrahim A, Bahechar N, Boukind EH: The Marjolin's ulcer, destiny of an unstable scar: about 54 cases of burn's sequalae. Ann Chir Plast Esthet 2002, 47(4):291-297.

21. Aydogdu E, Yildirim S, Akoz T: Is surgery an effective and adequate treatment in advanced matjolin'sulcer. Burns 2005, 31:421-31.

22. Hahn SB, Kim DJ, Jeon CH: Clinical study of Marjolin's ulcer. Yonsei Med 1990, 31(3):234-41.

23. Fishman RAJ, Parker MG: Malignancy and chronic wounds: Marjolin's Ulcer. J Burn Care Rehabil 1991, 12:218-23.

24. Hill BB, Sloan DA, Lee EY, McGrath PC, Kenady DE: Marjolin's ulcer of the foot caused by non-burn trauma. South Med J 1996, 89(7):707-10.

25. Neuman Z, Ben-Hur N, Shulman J: Trauma and skin cancer: implantation of epidermal elements and possible cause. Plast Reconstr Surg 1963, 32:649-656.

26. Bostwick J, Pendergrast WJ, Vasconez LO: Marjolin's ulcer: an immunologically priviledged tumor? Plast Reconstr Surg 1975, 57:66-69.

27. Trent JT, Kirsner RS: Wounds and malignancy. Advances in Skin and Wound Care 2003, 16:31-34.

28. Copcu E, Aktas A, Sismant N, Oztan Y: Thirty-one cases of Marjolin's ulcer. Clinical and Experimental Dermatology 2003, 28:138-41.

29. Lee SH, Shin MS, Kim HS: Somatic mutations of Fas (Apo-1/CD95) gene in cutaneous cell carcinomas arising from a burn scar. Journal of Investigative Dermatology 2000, 114(1):122-126.

30. Hayashi M, Tamura G, Kato N, Ansai S, Kondo S, Motoyama T: Genetic analysis of cutaneous squamous cell carcinomas arising from different areas. Patholology International 2003, 53(9):602-607.

31. Czarnecki D, Nicholson I, Tait B, Nash C: HLA DR4 is associated with the development of multiple basal cell carcinomas and malignant melanoma. Dermatolgica 1993, 187:16-18.

32. Harland $\mathrm{DL}$, Robinson WA, Franklin WA: Deletion of the $\mathrm{p} 53$ gene in a patient with aggressive burn scar carcinoma. J Trauma 1997, 42:104-107.

33. Gupta SK, Sandhir RK, Jaiswal AK, Kumar S: Marjolin's ulcer of the scalp invading calvarial bone, dura and brain. J Clin Neurosci 2005, 12:693-696.

34. Tutela RR Jr, Granick M, Benevenia J: Marjolin's ulcer arising in pressure ulcer. Adv Skin Wound Care 2004, 17:462-7.

35. Kowal-Vern A, Criswell BK: Burn scar neoplasms: a literature review and statistical analysis. Burns 2005, 31:403-13.

36. Arons MS, Rodin AE, Lynch JB, Lewis SR, Blocker TG Jr: Scar tissue carcinoma: II. An experimental study with special reference to burn scar carcinoma. Ann Surg 1966, 163:445-60.

37. Lawrence EA, Moore TC: Carcinoma arising in the scars of thermal burns, with special reference to the influence of the age at burn on the length of the induction period. Surg Gynecol Obstet 1952, 95:579-90.

38. Novick M, Card DA, Hardy SB, Spira M: Bum scar carcinoma: a review and analysis of 46 cases. J Trauma 1977, 17:809-17.
39. Celik E, ErogSlu S, KaracaogSlan N, Uzunismail A: Case report: Early arising Marjolin's ulcer in the scalp. Ann Burns Fire Disasters 2003, 16:217-21.

40. Malheiro E, Pinto A, Choupina M: Marjolin's ulcer of the scalp: case report and literature review. Ann Burns Fire Disasters 2001, 14:39-42.

41. Gamatsi E, McCulloch TA, Bailie FB, Srinivasan JR: Malignant melanoma in a skin graft: burn scar neoplasm or a transferred melanoma. Br J Plast Surg 2000, 53:342-52

42. Kontochristopoulos G, Kyriakis K, Symeonidou S, Katsiboulas V, Aroni K, Panteleos D, Katsambas A: Squamous cell carcinoma in chronic trophic ulcers of leprosy patients. J Eur Acad Dermatol Venerol 2000, 14:222-36.

43. Baldursson BT, Hedblad MA, Beitner $H$, Lindelöf B: Squamous cell carcinoma complicating chronic venous leg ulceration: a study of the histopathology, course and survival in 25 patients. Br J Dermatol 1999, 140:1148-52.

44. Eastman AL, Erdman WA, Lindberg GM, Hunt JL, Purdue GF, Fleming JB: Sentinel lymph node biopsy identifies occult nodal metastases in patients with Marjolin' ulcer. J Burn Care Rehabil 2004, 25(3):241-5.

doi:10.1186/1477-7819-10-38

Cite this article as: Chalya et al:: Marjolin's ulcers at a university teaching hospital in Northwestern Tanzania: a retrospective review of 56 cases. World Journal of Surgical Oncology 2012 10:38.

\section{Submit your next manuscript to BioMed Central and take full advantage of:}

- Convenient online submission

- Thorough peer review

- No space constraints or color figure charges

- Immediate publication on acceptance

- Inclusion in PubMed, CAS, Scopus and Google Scholar

- Research which is freely available for redistribution

Submit your manuscript at www.biomedcentral.com/submit
C) Biomed Central 